\title{
Low Temperature Formation of Silver and Silver-Copper Alloy Nano-Particles Using Plasma Enhanced Hydrogenation and Their Optical Properties
}

\author{
Zeinab Kiani, Yaser Abdi, Ezatollah Arzi* \\ Nanophysics Laboratory, Department of Physics, University of Tehran, Tehran, Iran \\ Email: *arzi@khayam.ut.ac.ir
}

Received November 22, 2011; revised January 12, 2012; accepted May 23, 2012

\begin{abstract}
In this paper, a novel method of producing nanoparticles at low temperatures using hydrogen bombardment of thin films, deposited on glass substrates, is introduced. Silver nanoparticles were obtained by this method in our Plasma Enhanced Chemical Vapor Deposition system. Optical and morphological characteristics of these nanoparticles were extensively studied for various conditions of plasma treatment, such as plasma power density, temperature, duration of hydrogen bombardment, thickness of the initial thin metallic film etc. In addition, Ag-Cu alloy nanoparticles on glass substrates were also achieved. The process of nanoparticle formation in this method shows that several kinds of metals and semiconductors nanoparticles can be obtained using this approach. Scanning Electron Microscopy, Atomic Force Microscopy and Transmission Electron Microscopy were used to analyze the nanostructures.
\end{abstract}

Keywords: Nanoparticles; Plasma; Hydrogen; PECVD; Silver; Alloy

\section{Introduction}

Because of their linear and nonlinear optical properties, metal nanoparticles have been widely studied in recent years. They are suitable candidates for various devices because of their unique optical [1], electronic [2], catalysis [3], chemical [4] properties. Many different methods were applied for producing nanoparticles such as chemical synthesis [5], exploding wire [6], and laser ablation [7]. We have used a novel different method that produces nanoparticles at a temperature much lower than the melting point of their respective bulk using hydrogen plasma in Enhanced Chemical Vapor Deposition system. In this work, silver nanoparticles and bimetallic alloy of silver-copper nanoparticles were produced by this plasma treatment method. This method can also be used for the formation of other metallic or semiconductors nanoparticles by PVD method on glass or silicon or other substrates, out of which two examples of nickel and silicon nanoparticles are presented.

Optical properties of metal nanoparticles are related to the excitation of surface plasmon. The parameters such as size and shape [8,9], surrounding medium [10], metallic species [11] of nanoparticles change the excitation energy of surface plasmons. We have investigated the effects of size on the absorption spectra of the nanoparti-

${ }^{*}$ Corresponding author. cles.

\section{Experimental}

The work started by cleaning the glass substrate in the standard RCA1 solution, which contained $\mathrm{NH}_{3} /$ $\mathrm{H}_{2} \mathrm{O}_{2} / \mathrm{DI}-\mathrm{H}_{2} \mathrm{O}$ with relative volume proportions of $1: 1: 5$, and followed by the deposition of a thin layer of silver with the thickness of about $10 \mathrm{~nm}$ in a thermal evaporation system at the base pressure of $5 \times 10^{-6}$ torr [Formation of bimetallic alloy nanoparticles needs to evaporate two metallic sources simultaneously in the physical vapor deposition (PVD) system]. The silver-coated glass was then put in the direct current plasma enhanced chemical vapor deposition (DC-PECVD) apparatus, as shown schematically in Figure 1. This DC-PECVD setup has two flat electrodes, made of iron, which hydrogen plasma forms between them by the application of a voltage between the electrodes. The sample was placed on the cathode (lower electrode) and during the plasma treatment was bombarded by hydrogen radicals. We made an electrical connection between the metallic layer and the cathode to prevent the build up of positive charges on glass substrate that might repel the plasma. The substrate temperature was varied between $200^{\circ} \mathrm{C}$ and $400^{\circ} \mathrm{C}$ and the hydrogen pressure was maintained at 0.4 2 torr during the Plasma treatment in the DC-PECVD 


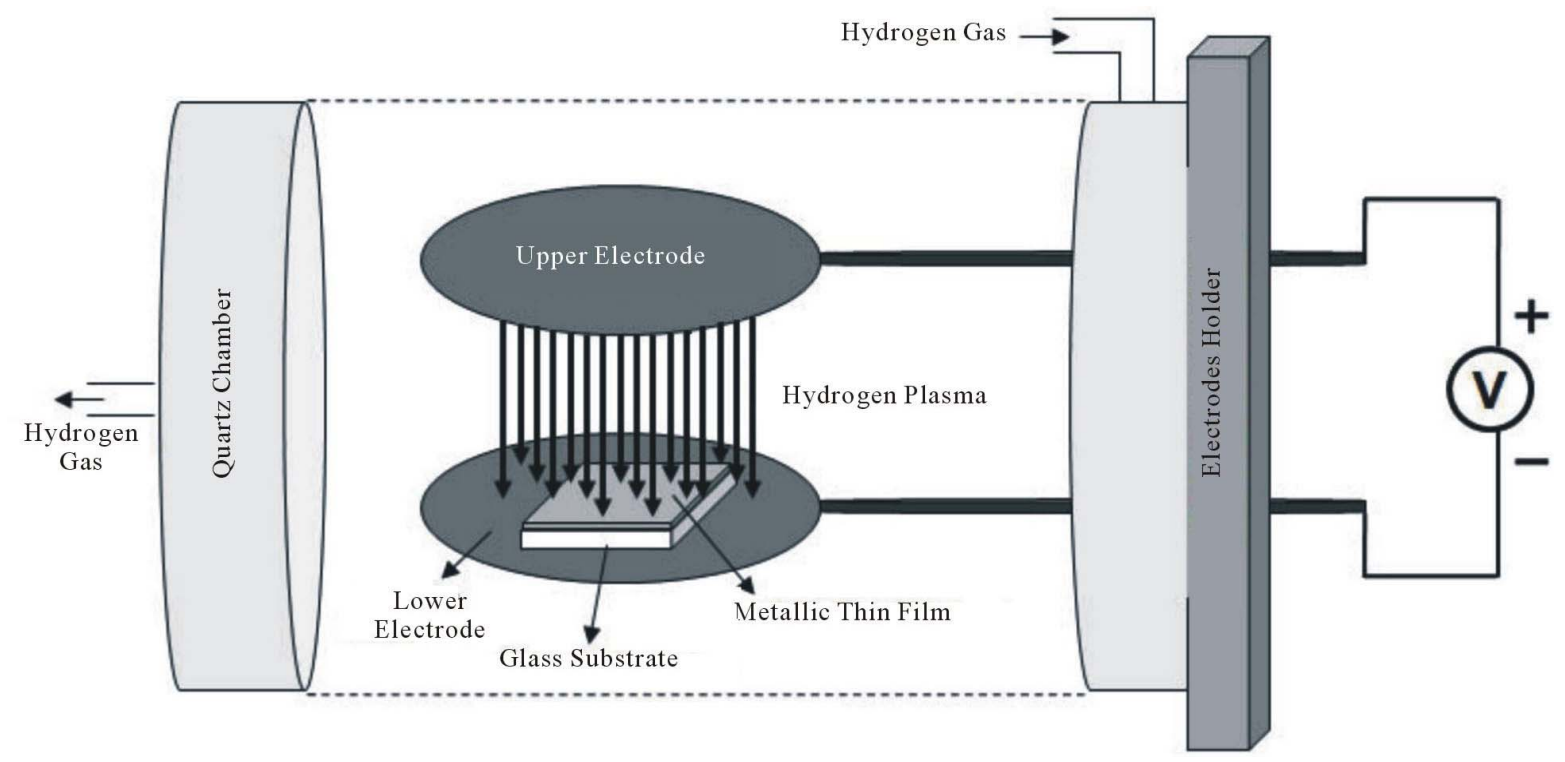

Figure 1. Schematic diagram of DC-PECVD chamber showing two electrodes being connected to a voltage supplier. The rest of the apparatus including the heater, the temperature control, the inlets and the outlets etc are not shown.

system. The flow rate of hydrogen was kept between 50 to $70 \mathrm{Sccm}$. The power density of the DC-PECVD reactor during the hydrogen bombardment step was varied between 100 and $800 \mathrm{~mW} / \mathrm{cm}^{2}$. The subsequent annealing step was conducted in situ at a substrate temperature of $50^{\circ} \mathrm{C}$ higher than what had been used for the bombardment step. These successive plasma treatment/annealing steps were carried out for each sample. Based on the investigation conducted in this study, the bombardment step served to etch the deposited layer, forming nano-clusters and nucleation sites for subsequent formation of the nanoparticles. However, it was observed that if the duration of the hydrogen bombardment step exceeded a certain time, the entire deposited layer was removed. During the annealing period, the hydrogen trapped in the layer was ejected out and some energy was imparted to the nano-structures, enhancing the chance of formation of the nanoparticles. It should be mentioned that this step has been performed in the absence of hydrogen plasma. On the other hand, it has been observed that if the annealing step is carried out for longer periods, the small grains of nanoparticles merge, forming larger grains. The sequence of consecutive hydrogen bombardment and annealing steps has been found to be an optimal condition for the evolution of nanoparticles.

Duration and temperature of annealing and bombardment steps, plasma power, and the thickness of the initial thin metallic film influence the size of nanoparticles. We have investigated the effects of such parameters on nanoparticles size and the absorption spectra. Scanning electron microscope and atomic force microscope were used to analyze the surface morphology of the as prepared samples.

\section{Results and Discussion}

Figure 2 includes four atomic force microscope (AFM) and two scanning electron microscope (SEM) images of as-prepared silver and silver-copper nanoparticles demonstrating surface morphology and nanostructures of the samples prepared at different condition of nanoparticles formation. As shown in this figure well-isolated nanoparticles with different diameter size were obtained. The preparation condition and the final average size of each sample are given in Table 1. The thickness of the initial layer for Ag samples (a-d) was about $10 \mathrm{~nm}$ and for $\mathrm{Ag} / \mathrm{Cu}$ alloy samples (e \& f) was about $16 \mathrm{~nm}$, which contained $70 \% \mathrm{Cu}$ and $30 \% \mathrm{Ag}$ by weight. During the plasma treatment the deposited layer was transformed to nano-islands. This reshaping (from flat layer to spherical particles) leads to have nanoparticles with diameters larger than the layer thickness. It is worth mentioning that the total volumes of the deposited material before and after the plasma treatment are the same.

For investigating the effects of temperature, plasma power and other parameters of plasma treatment on the structure and optical properties of nanoparticles, we have prepared quite a number of samples, the results of which are as follows. Figure 3 shows AFM images and absorption spectra of two $\mathrm{Ag}$ samples prepared at different temperatures of bombardment step, i.e. $300^{\circ} \mathrm{C}$ and $380^{\circ} \mathrm{C}$. It can be clearly seen that the higher the temperature, the larger the nanoparticles. In addition, Figure 3(c) shows that higher temperature shifts absorption spectra peak towards longer wavelengths. It is worth mentioning that the duration and plasma power density have been maintained at 20 minutes and $100 \mathrm{~mW} / \mathrm{cm}^{2}$ during the preparation step. 
Figure 4 is a comparison between the samples prepared at two different plasma power densities of 100 and 600 $\mathrm{mW} / \mathrm{cm}^{2}$. As shown in this figure, at lower plasma power density during the bombardment, smaller nanoparticles

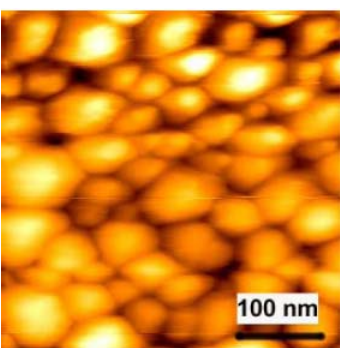

(a)

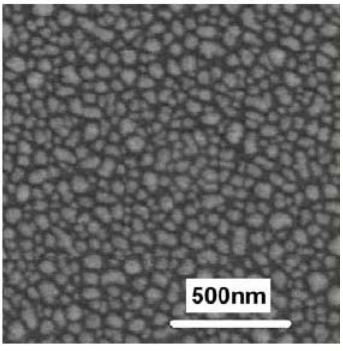

(c)

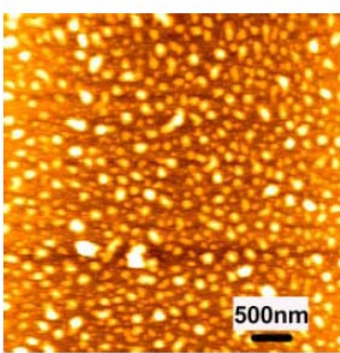

(e)

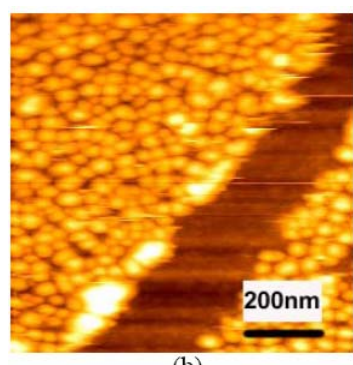

(b)

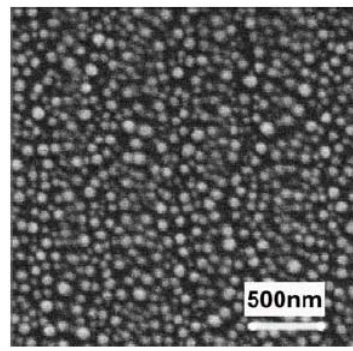

(d)

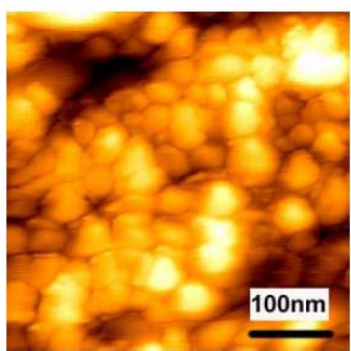

(f)
Figure 2. AFM and SEM images of silver nanoparticles (a)-(d) and Ag/Cu alloy samples ((e) \& (f)) formed on glass substrates using hydrogen plasma treatment, showing that different conditions of sample preparation lead to different sizes of nanoparticles. are achieved and correspondingly, the absorption peak appears at a wavelength shorter than that of the sample prepared at higher plasma power density. Thus, we see that changes in morphology and sizing of nanoparticles caused by temperature and plasma power density lead to variations in their optical properties. It seems that smaller particles have their absorption peak at shorter wavelengths range. We have also investigated the effects of other ranges of plasma power density on our samples. It is observed that raising the power density to values above $800 \mathrm{~mW} / \mathrm{cm}^{2}$ will lead to total etching of the deposited layer. On the other hand, no grain formation was observed at power densities below $100 \mathrm{~mW} / \mathrm{cm}^{2}$, as confirmed by SEM and AFM results.

Comparison between two samples, one of them bombarded for 20 minutes and the other one for 45 minutes but both at the same temperature of $300^{\circ} \mathrm{C}$ with the same plasma power density of $150 \mathrm{~mW} / \mathrm{cm}^{2}$, is represented in Figure 5. This comparison demonstrates that longer duration of bombardment results in smaller nanoparticles and hence the absorption spectrum gets blue shifted. Further experiments revealed that the thickness of the initial deposited layer is very important. It is found that increasing the layer thickness above $50 \mathrm{~nm}$ leads to observe no grain formation. Figure 6 shows SEM images of three samples having silver layer thickness of 70, 40 and $10 \mathrm{~nm}$ prepared at the same condition of plasma treatment and annealing step, confirming the above mentioned conclusion. In addition, Figure 6(d) shows absorption spectra of these samples showing no absorption peak in the spectrum of the sample with $70 \mathrm{~nm}$ initial layer thickness.

We have used this approach not only to produce silver nanoparticles, but also for other materials such as nickel and silicon nanoparticles, their SEM images are shown in Figure 7. Thus we believe that this method is suitable for producing nanoparticles of various metallic and semiconductor materials.

Table 1. Sample preparation conditions for Figure 2.

\begin{tabular}{|c|c|c|c|c|}
\hline \multirow{2}{*}{ Average particle size (nm) } & \multirow{2}{*}{ Plasma power density $\left(\mathrm{mW} / \mathrm{cm}^{2}\right)$} & \multicolumn{2}{|c|}{ Bombardment condition } & \multirow{2}{*}{ Sample } \\
\hline & & Temperature $\left({ }^{\circ} \mathrm{C}\right)$ & Duration (minutes) & \\
\hline 75 & 200 & 300 & 15 & $\mathrm{a}$ \\
\hline 50 & 180 & 300 & 20 & $\mathrm{~b}$ \\
\hline 65 & 230 & 300 & 20 & ${ }^{*} \mathrm{c}$ \\
\hline 70 & 300 & 380 & 20 & $\mathrm{~d}$ \\
\hline 145 & 120 & 220 & 20 & e \\
\hline 55 & 130 & 300 & 15 & $\mathrm{f}$ \\
\hline
\end{tabular}

\footnotetext{
*It was then annealed at $400^{\circ} \mathrm{C}$ for 20 minutes.
} 


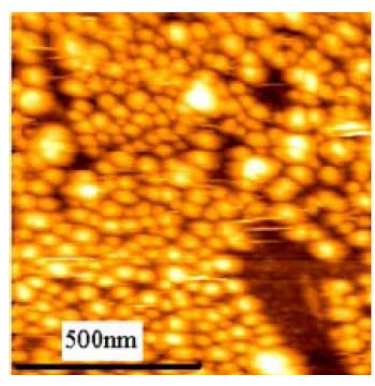

(a)

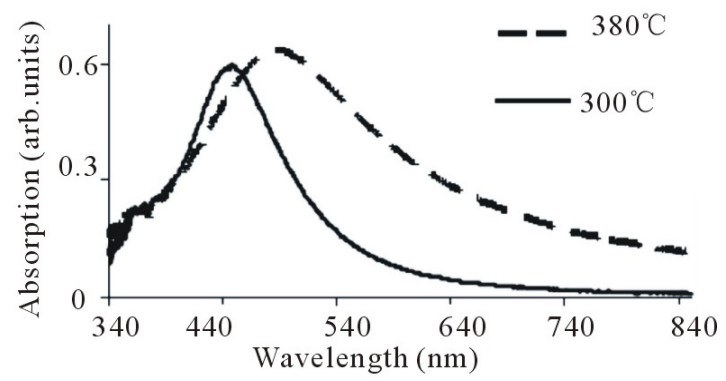

(c)

Figure 3. AFM images of two Ag samples prepared at different temperature of the bombardment step, (a) $300^{\circ} \mathrm{C}$ and (b) $380^{\circ} \mathrm{C}$; (c) Absorption spectra of the two above-mentioned samples.
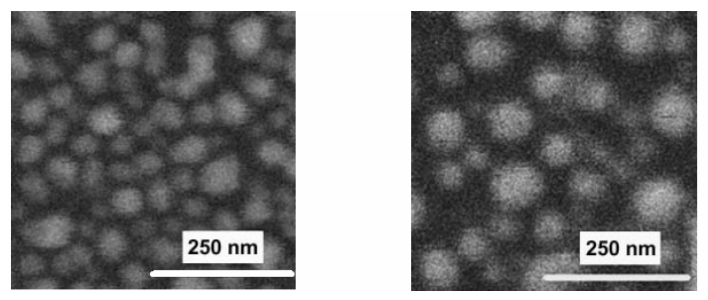

(a)

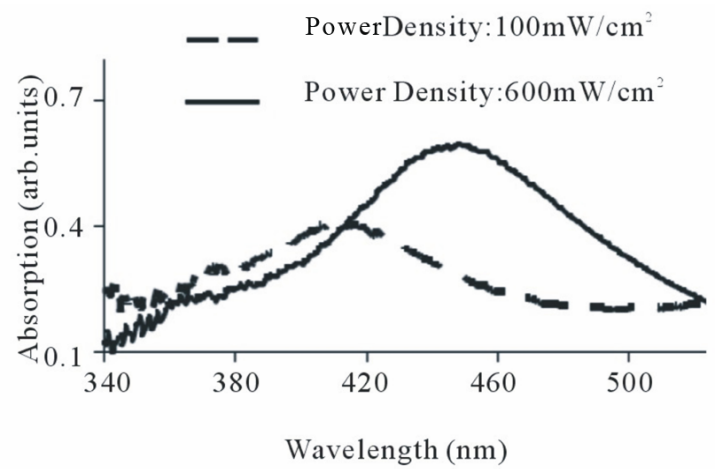

(b)

Figure 4. (a) SEM images of the above-mentioned samples, formed at plasma power density of $100 \mathrm{~mW} / \mathrm{cm}^{2}$ (left) and $600 \mathrm{~mW} / \mathrm{cm}^{2}$ (right). These SEM images and the corresponding absorption spectra show that lower power density of plasma during bombardment leads to the formation of smaller nanoparticles; (b) Absorption spectra of the Ag samples that have been formed at the plasma power density of 100 and $650 \mathrm{~mW} / \mathrm{cm}^{2}$.

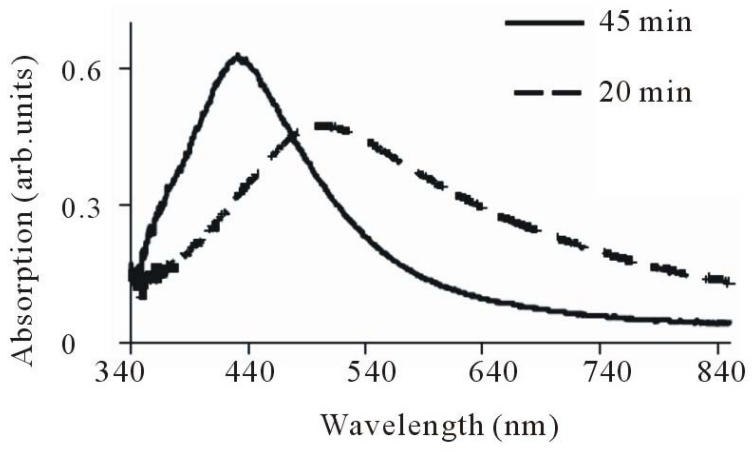

Figure 5. Absorption spectra of samples having bombarded for different periods: 20 and 45 minutes. Comparison between them shows that longer time of bombardment leads to form smaller nanoparticles.

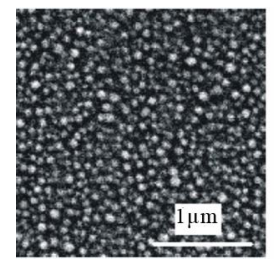

(a)

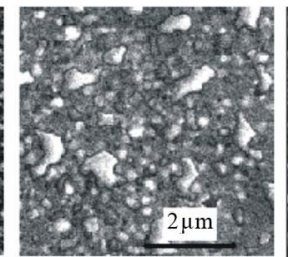

(b)

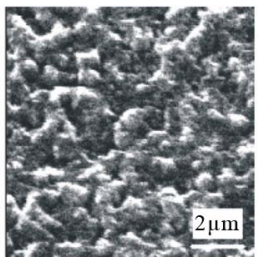

(c)

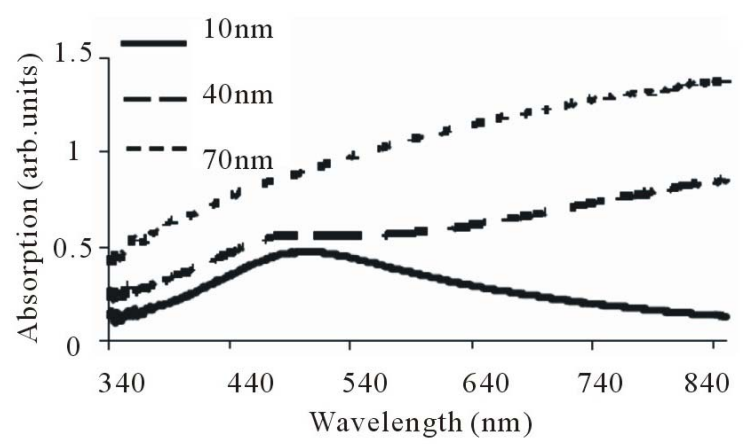

(d)

Figure 6. SEM images of silver samples prepared on silicon substrates at the same condition of bombardment treatment, except that the initial thickness of deposited layer was (a) $10 \mathrm{~nm}$; (b) $40 \mathrm{~nm}$ and (c) $70 \mathrm{~nm}$; (d) Absorption spectra of the above mentioned samples.

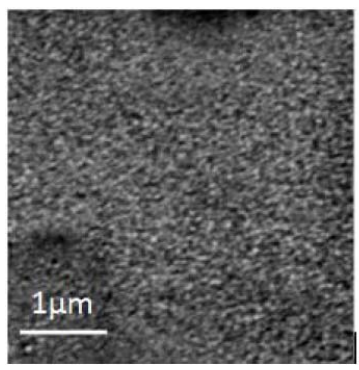

(a)

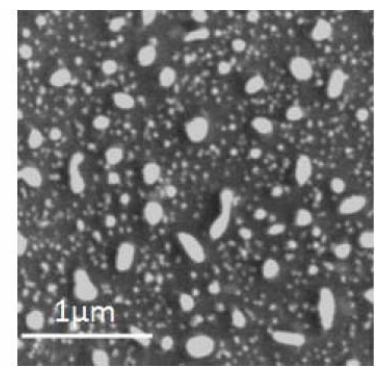

(b)
Figure 7. SEM images of (a) semiconductor silicon nanoparticles; (b) metallic nickel nanoparticles, produced by the proposed hydrogen treatment method. 
To form alloy nanoparticles, silver and copper were simultaneously evaporated by PVD system as two components of bimetallic alloy on glass substrate. AFM images of plasma treated silver/copper nanoparticles are represented in Figure 8. Figure 9 shows TEM images of silver/copper alloy nanoparticles that were produced by this novel hydrogen bombardment method, which clearly shows the formation of alloy nanoparticles. By looking carefully at this image, one can see that some parts of some grains in the TEM image are darker, which means that these grains contain the two component materials and thus the alloy of silver/copper was formed in this sample. Therefore, the method is capable of producing alloys. Another method for depicting the structure is the optical analysis [12]. Optical results of the as-pre- pared samples depict two different structures for these nanostructures. Figure 10 shows the absorption spectra of two $\mathrm{Ag} / \mathrm{Cu}$ nanoparticles prepared at two different temperatures of $200^{\circ} \mathrm{C}$ and $300^{\circ} \mathrm{C}$ during the bombardment step. As shown in this figure, the sample prepared at the lower temperature has two isolated peaks corresponding to the absorption peaks of pure silver and pure copper nanoparticles whilst the other one (dashed curve) has a single

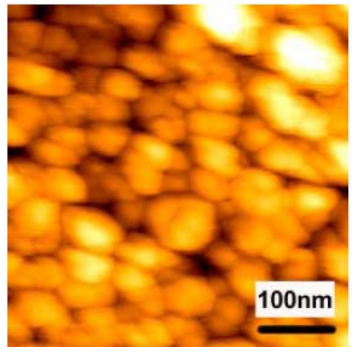

(a)

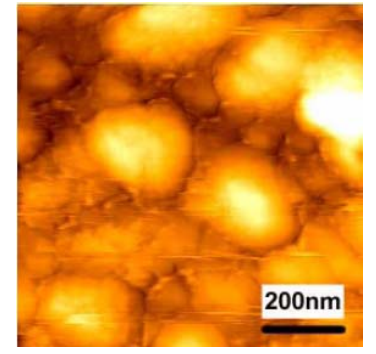

(b)
Figure 8. AFM images of Ag/Cu alloy nanoparticles with initial layer thickness of about $15 \mathbf{~ n m}$ that have been prepared in different condition, (a) contains 30\% Ag and $70 \%$ $\mathrm{Cu}$ by weight, bombarded for 20 minutes at plasma power density of $100 \mathrm{~mW} / \mathrm{cm}^{2}$ at $300{ }^{\circ} \mathrm{C}$ and (b) contains $50 \% \mathrm{Ag}$ and $50 \% \mathrm{Cu}$ by weight, bombarded for 20 minutes at plasma power density of $140 \mathrm{~mW} / \mathrm{cm}^{2}$ at $370^{\circ} \mathrm{C}$.
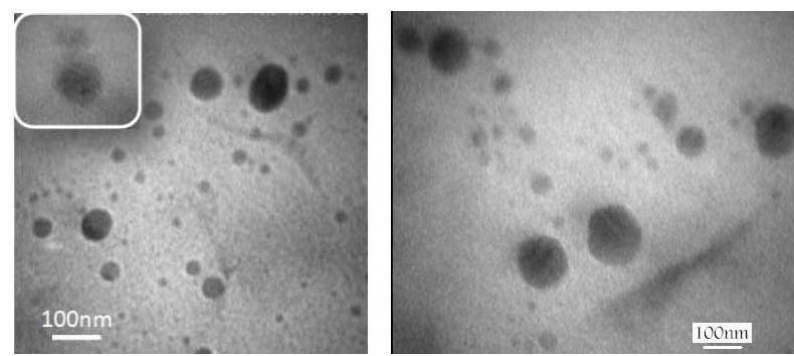

Figure 9. TEM images of silver/copper alloy nanoparticles showing that the grains were formed by this method. The darker color of some parts of the grains shows that the grains contain more than one material, and thus the silver/copper alloy nanoparticles were formed. peak at a wavelength between the two above-mentioned peaks. It depicts that the sample with two peaks is just a mixture of $\mathrm{Ag}$ nanoparticles and $\mathrm{Cu}$ nanoparticles but the other one is an alloy of $\mathrm{Ag} / \mathrm{Cu}$ nanoparticles. Figure 11 shows the absorption spectra of the alloys with different mixing ratio. As shown in this figure, shifting the absorption peak towards the blue or red regions depends on the mass percentage of silver or copper components in the alloy.

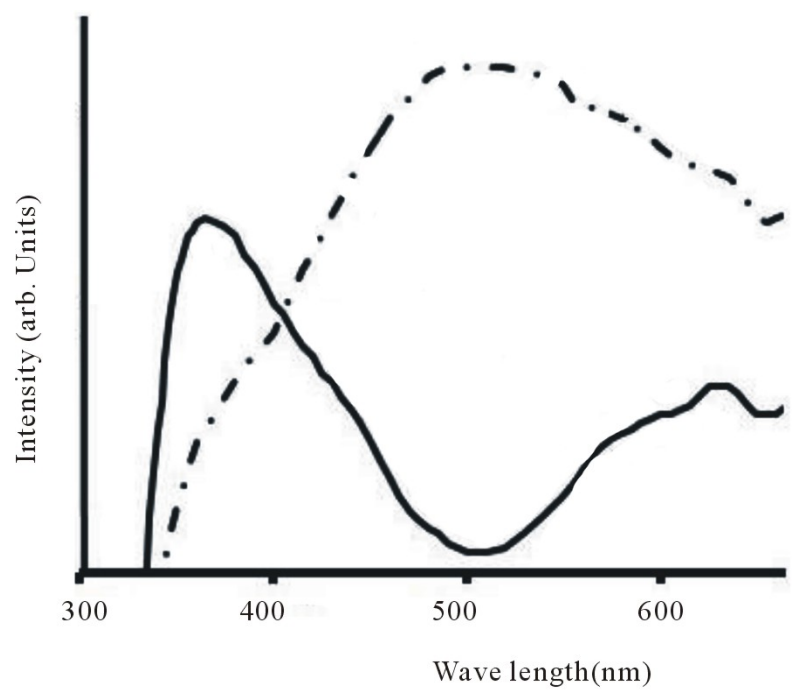

Figure 10. Absorption spectra of samples having different temperature in their bombardment steps. The sample that exhibits two peaks in its absorption spectrum (solid line), bombarded at $200^{\circ} \mathrm{C}$, shows the formation of separated $\mathrm{Ag}$ and $\mathrm{Cu}$ nanoparticles, not alloy. The other sample, which is bombarded at $300^{\circ} \mathrm{C}$, shows one peak in its spectrum (dashed line) that can be attributed to alloy formation.

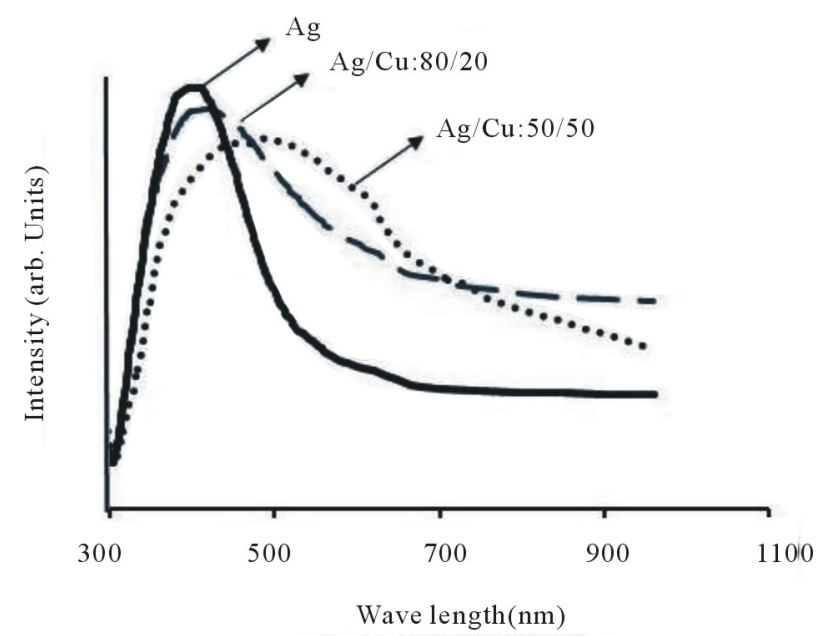

Figure 11. Comparison between the absorption spectra of $\mathrm{Ag} / \mathrm{Cu}$ alloy nanoparticles having different weight ratio of $\mathrm{Cu}$; all prepared at the same condition. It shows that by increasing the $\mathrm{Cu}$ ratio in the nanoparticles, the absorption spectrum gets a red shift. 
The results of the visible light spectroscopy presented in this paper show the wavelength dependent absorption in the fabricated nanostructures. It can be explained by the Langmuir theory describing the collective oscillations of surface charges. In metal particles with subwavelength dimensions there are in fact dipole electron oscillations bounded by the nanoscopic particle. From quantum mechanical point of view, the charge oscillations are easily excited by the incident photons with energy of $\hbar \omega_{\mathrm{p}}$, where $\omega_{\mathrm{p}}$ is the resonance frequency of the charge oscillations depends on the size, material and shape of the nanoparticle. The source of absorption peaks in the presented spectra is the light induced quantum excitation of such oscillations.

\section{Conclusion}

In summary, we have successfully produced the silver and silver-copper alloy nanoparticles using a low temperature plasma bombardment method, well below the melting point of the sample constituents. The method can be used for any other materials to produce nanoparticles of them on any arbitrary substrates (even flexible substrates). It is easy to make a patterned structure of nanoparticles by this method. We believe that the proposed method can produce clusters of nanoparticles and may be the produced films can be used in device fabrications such as transistors, single electron transistors, nanoparticles-based gas sensors etc. Optical measurements of as-prepared nanoparticles confirm the quantum behavior of the samples arising from decreasing the size and confinement.

\section{Acknowledgements}

We would like to thank the research council of the University of Tehran for partial financial support.

\section{REFERENCES}

[1] W. H. Weber and G. W. Ford, "Propagation of Optical Excitations by Dipolar Interactions in Metal Nanoparticle Chains," Physical Review B, Vol. 70, No. 12, 2004, pp. 125429.1-125429.8. doi:10.1103/PhysRevB.70.125429

[2] S. Link and M. A. El-Sayed, "Spectral Properties and Relaxation Dynamics of Surface Plasmon Electronic Oscillations in Gold and Silver Nanodots and Nanorods," The Journal of Physical Chemistry B, Vol. 103, No. 40,
1999, pp. 8410-8426. doi:10.1021/jp9917648

[3] W. P. Zhou, A. Lewera, R. Larsen, R. I. Masel, P. S. Bagus and A. Wieckowski, "Size Effects in Electronic and Catalytic Properties of Unsupported Palladium Nanoparticles in Electrooxidation of Formic Acid," The Journal of Physical Chemistry B, Vol. 110, No. 27, 2006, pp. 13393-13398. doi:10.1021/jp061690h

[4] P. Waszczuk, T. M. Barnard, C. Rice, R. I. Masel and A. Wieckowski, "A Nanoparticle Catalyst with Superior Activity for Electrooxidation of Formic Acid," Electrochemistry Communications, Vol. 4, No. 7, 2003, pp. 599603. doi:10.1016/S1388-2481(02)00386-7

[5] B. Choi and H.-H. Lee, "Characterization of the Optical Properties of Silver Nanoparticle Films," Nanotechnology, Vol. 18, No. 7, 2007, Article ID: 075706.

[6] T. K. Sindhu, R. Sarathi and S. R. Chakravarthy, "Understanding Nanoparticle Formation by a Wire Explosion Process through Experimental and Modeling Studies," Nanotechnology, Vol. 19, No. 2, 2008, Article ID:. 025703.

[7] S. H. Ko and Y. Choi, "Nanosecond Laser Ablation of Gold Nanoparticle Films," Applied Physics Letters, Vol. 89, No. 14, 2006, p. 141126. doi:10.1063/1.2360241

[8] M. Valden, X. Lai and D. W. Goodman, "Onset of Catalytic Activity of Gold Clusters on Titania with the Appearance of Nonmetallic Properties," Science, Vol. 281, No. 5383, 1998, pp. 1647-1650. doi:10.1126/science.281.5383.1647

[9] X. Y. Xu, K. K. Caswell, E. Tucker, S. Kabisatpathy, K. L. Brodhacker and W. A. Scrivens, "Size and Shape Separation of Gold Nanoparticles with Preparative Gel Electrophoresis," Journal of Choromatography A, Vol. 1167, No. 1, 2007, pp. 35-41. doi:10.1016/j.chroma.2007.07.056

[10] N. Nath and A. Chilkoti, "A Colorimetric Gold Nanoparticle Sensor to Interrogate Biomolecular Interactions in Real Time on a Surface," Analytical Chemistry, Vol. 74, No. 3, 2002, pp. 504-509. doi:10.1021/ac015657x

[11] K. Esumi, T. Matsumoto, Y. Seto and T. Yoshimura, "Preparation of Gold-, Gold/Silver-Dendrimer Nanocomposites in the Presence of Benzoin in Ethanol by UV Irradiation," Journal of Colloid and Interface Science, Vol. 284, No. 1, 2005, pp. 199-203. doi:10.1016/j.jcis.2004.09.020

[12] H. J. Jiang, K. Moon and C. P. Wong, "Synthesis of $\mathrm{Ag}-\mathrm{Cu}$ Alloy Nanoparticles for Lead-Free Interconnect Materials," Proceedings of International Symposium on Advanced Packaging Materials: Processes, Properties and Interfaces, Irvine, 16-18 March 2005, pp. 173-177. doi:10.1109/ISAPM.2005.1432072 